\title{
Satellite PHY-layer Selector Design for Video Applications in Tropical Areas
}

\author{
David Pradas, Lei Jiang, M.A. Vázquez Castro \\ Dpt. Telecom. and Systems Engineering \\ Universitat Autònoma de Barcelona (UAB), Barcelona, Spain \\ david.pradas@uab.es, jiang.lei@uab.es, angeles.vazquez@uab.es
}

\author{
Paolo Barsocchi, Francesco Potortì \\ ISTI \\ CNR Research Area, Pisa, Italy \\ paolo.barsocchi@isti.cnr.it, potorti@isti.cnr.it
}

\begin{abstract}
This paper aims at providing different cost-efficient solutions for the channel impairments in tropical areas. In order to extend service to isolated areas, we propose an hybrid architecture based on DVB-S2/RCS + Wi-Fi networks. In this scenario, the delay of the ACM reaction to fade changes can affect the quality of the video transmission, especially because of the characteristics of tropical deep fading events. In order to avoid QoS reduction, we focus on the DVB PHY-layer shifted threshold, which we study not only for different Amazon areas and different rain conditions, but also for different reaction delays. The performance of the PHY-layer selector is evaluated in terms of video quality (PSNR), packet error rate and bandwidth efficiency.
\end{abstract}

\section{INTRODUCTION}

The demand for broadband connectivity in low-densely populated areas has given rise to a big interest in hybrid networks, especially those composed of a satellite backhaul and a wireless terrestrial network. In this paper, we focus on a DVB-S2/RCS system with Adaptive Coding and Modulation (ACM) for the satellite link and a Wi-Fi network for the indoor terrestrial wireless link. Figure 1 depicts the considered scenario, which reproduces the hybrid network used to give service to small villages along the Amazon River [1]. The video stream is transmitted by the video stream server to the satellite transmitter, which broadcasts it to the satellite network. Each satellite receiver broadcasts the video stream to a terrestrial indoor wireless network. For the Amazon scenario considered here, the satellite link is the bottleneck of the system in terms of capacity, because the users in the indoor $\mathrm{Wi}$ Fi network are fixed; consequently, the terrestrial link condition has no impact on the overall conditions of the hybrid channel.

In a previous paper [2], we show that during the rain event, the delay of the ACM reaction to fade changes can affect the Quality of Service (QoS) of a multicast video streaming. Specifically, there is a noticeable delay from the moment an RCS Terminal (RCST) detects an ongoing fade event to the moment it receives from the Network Control Center (NCC) the transmission with the modulation and coding rates appropriate to cope with the increased atmospheric attenuation. We demonstrated that a shifted threshold and Link Layer FEC (LL-FEC) are useful to compensate for the packet errors introduced by the ACM switching delay, thus allowing the transmission of good video quality. An appropriate choice of the threshold can achieve almost the same PER reduction as using the strongest high layer codes, but with a higher bandwidth efficiency gain

This work aims at demonstrating the QoS improvement of the video transmitted in the hybrid network obtained by optimising the shifted threshold offset and shows how to make efficient use of bandwidth in order to reduce costs in Amazon areas. In order to improve accuracy in the results obtained, we will take into account the allocation delay and an extensive set of rain events (including time and space diversity).

The main parameters evaluated for this purpose are the packet error rate, the delay, and the bandwidth overhead due to the use of the proposed technique. Finally, the received video will be evaluated by using the PSNR (Peak Signal to Noise Ratio) metric.

The paper is organised as follows. The hybrid scenario, based on the combination of DVB-S2 as the satellite backhaul and $\mathrm{Wi}-\mathrm{Fi}$ as the terrestrial link, is presented in section II. An extended description of the shifted threshold solution, including all related configuration parameters, is carried out in section III. The simulation platform is presented in section IV, following a detailed analysis of the results for different setting configurations focusing on the video quality evaluation. Finally, in the conclusions, we identify the achievements of the paper and the issues to be addressed in future works.

\section{System MODEL}

We consider a GEO (Geostationary Earth Orbit) Multibeam Broadband Satellite (MBS) link with a DVB-S2/RCS [4], [5] air interface. DVB-S2 incorporates ACM on a time slot basis, chosen on the base of the SNIR (Signal-to-Noise-plusInterference Ratio) at the destination terminal. The system implements a TDM/FDM (hybrid Time and Frequency Division Multiplexing) scheme; each time slot contains a DVB-S2 physical layer packet, with a constant amount of coded symbols but an ACM mode-dependent number of information bits and symbols: the consequence is a variable packet transmission time. By choosing 23 out of the 27 ACM modes proposed in the standard [4] we obtain coding gain increments between modes of about $1 \mathrm{~dB}$. We assume transmission in the $K_{a}$ band (20-30 GHz), where fading is strongly affected by rain events. The Wi-Fi access point is in charge of efficiently using the limited radio spectrum resources. On the satellite side, the RCST measures the down-link attenuation and signals it to 
the NCC, which in turn adapts the transmission parameters to the observed fading.

The combination of these two technologies is useful to enable connectivity in non-urban areas. It is one of the solutions that are envisaged in the context of the BRASIL project in order to provide different types of network access to villages along the Amazon River.

\section{SHIFTED THRESHOLD}

The physical layer selector of the RCST (see Annex E in [4]) estimates the optimal physical layer configuration (i.e. the ACM transmission mode) to be used in each channel condition. The Channel State Information (CSI) is sent to the NCC, which switches to the adequate ACM mode in order to protect data efficiently according to the channel variations. For fixed RCST, as is the case of our scenario, weather conditions are the main channel impairment.

In order to guarantee reliable and efficient system operation, the ACM selector shall account for: a) SNIR estimation errors, b) propagation delay and possible channel variations occurring between the user request and the application of the updated ACM mode.

According to [3], we assume that the RCST can send the CSI only once per superframe, and moreover, we consider that measurements are averaged during whole superframe in order to filter out the highest frequencies of the attenuation process. Specifically, the terminal takes measurement samples during superframe $i$, their average is sent at the beginning of superframe $i+1$, and NCC can use the correct modcod in superframe $i+3$. The delay can thus be computed as $R T T+2 \cdot S F_{d u r}$, where $S F_{d u r}$ is the super-frame length duration and RTT, the Round Trip Time (about $500 \mathrm{~ms}$ ). SNIR measures are exponentially averaged at each time $t_{i}$ using a decay constant of 0.5 . The number of measurements averaged depends on $S F_{d u r}$ and the CSI sampling time $t_{s}$, e.g. if $S F_{d u r}=800 \mathrm{~ms}$ and $t_{s}=250 \mathrm{~ms}$, the RCST can compute and average 3 attenuation measurements.

Our goal is to develop an algorithm for computing the shifted threshold that produces the best quality in the video transmission. The algorithm consists of shifting by a given margin (in $\mathrm{dB}$ ) the threshold of the standardised ACM modes of DVB-S2. Two are the main purposes of shifting the threshold, as specified in the standard: a) to avoid loss of data when attenuation is increasing, since the data is not correctly protected, b) to avoid extra payload and loss of efficiency when attenuation is decreasing, since the data is overprotected. Although the second point will be also studied in terms of the bandwidth efficiency, it is out of the scope of this paper, and our algorithm will focus on minimising the lost data. The shifted threshold is offset against the original threshold by a quantity that is generally dependent on the estimation error and the slope of the rain event. In this paper we focus on a fixed threshold offset for different types of tropical rain events. The results we obtain will guide us in the design and the implementation of an adaptive algorithm that we will consider for future works.

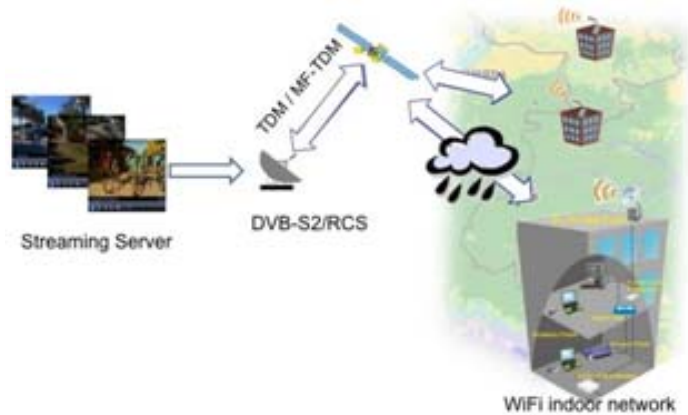

Fig. 1. Hybrid Architecture

\section{RESULTS}

\section{A. Simulation Platform}

A Multilayer Protection simulation platform has been developed in order to quickly assess the performance of different parameter configurations without repeating the time-consuming physical layer simulations. The ACM switch Physical-Layer module depicted in the upper-right corner of Fig. 2 generates the satellite channel attenuation time series and interfaces with the Link Layer simulator. The PHY settings module chooses the shifted threshold and the super-frame length. The simulator takes streams of IP packets as input and applies the MPE-FEC encoding technique, generating an MPEG-2 TS by encapsulating Multi-Protocol Encapsulation (MPE) sections and MPE-FEC sections. Then, MPE-sections are encapsulated in different BBFrames as specified in [4]. The output of the physical-layer simulator is used to mark the BBFrames as correctly received or being erroneous. After BBFrame and MPE-FEC decoding, the sequence of IP packets affected by the unreliable columns is obtained and the PER at the IP level is computed. Finally, PSNR is computed by comparing both videos; original and corrupted; when the decoder is incapable of decoding a video frame, the previous decoded frame is frozen until the decoder can recover from the stream corruption - normally at the next I frame starting a new GOP (Group Of Pictures).

\section{B. Parameters and Results}

In order to get a realistic estimation of the system's efficiency, we consider a significant amount of measured fading events, which have been selected from 1 month measurements at two different locations in the Amazon area (Tabatinga and Caxias, favourable and unfavourable case respectively). In particular, the considered satellite (AMAZONAS-1) operates from geostationary orbit, at the $61^{\circ} \mathrm{W}$ orbital position, and we focus on the $12 \mathrm{GHz} \mathrm{K}$ Band transponders.

A data rate of $530 \mathrm{~kb} / \mathrm{s}$ has been chosen to study the performance of the video streaming application. First of all, in Fig. 3, we evaluate performance in terms of PER versus the threshold offset, for two different locations in rain conditions: the Tabatinga area with maximum attenuation $a t t_{\max }$ of $16 \mathrm{~dB}$ and the Caxias area with $a t t_{\max } 9 \mathrm{~dB}$, plus a quasi-clear sky case with $a t t_{\max }$ around 2-3 $\mathrm{dB}$. We studied the performance for two $S F_{d u r}$ values. As expected, the PER is strongly 


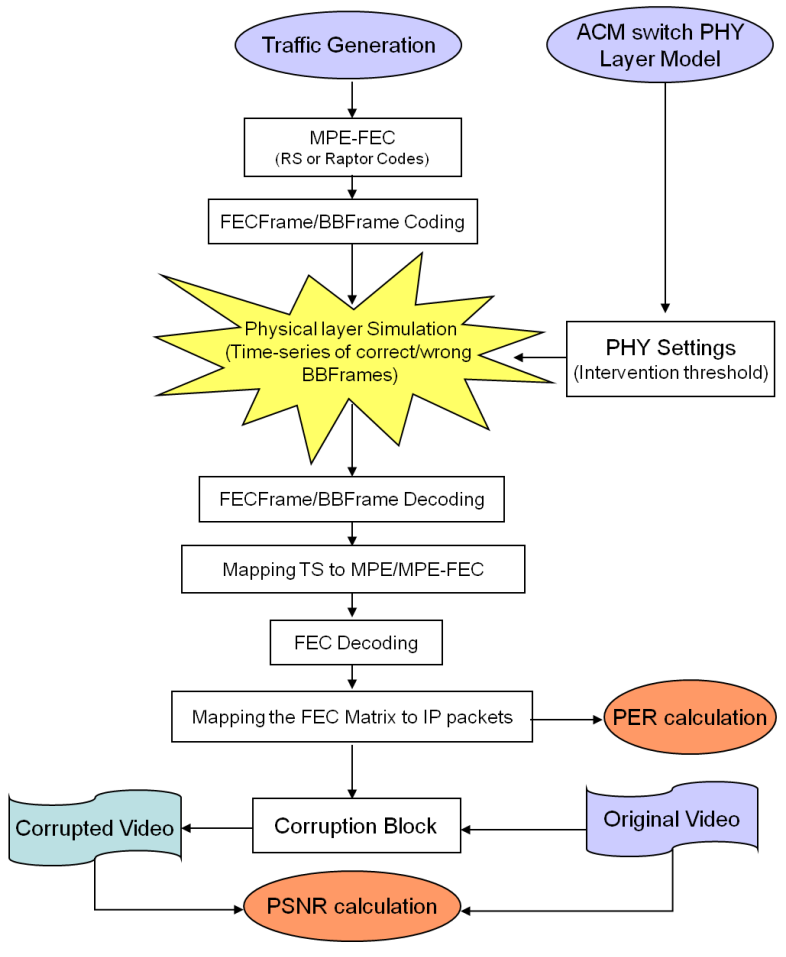

Fig. 2. Simulation Flow Diagram

affected by both the $S F_{d u r}$ and the location. By increasing the threshold offset, the PER is reduced: we observe more than $50 \%$ reduction for $0.2 \mathrm{~dB}$.

In order to make efficient use of bandwidth, we also investigate the trade-off between threshold offset and bandwidth efficiency in Fig. 4. A high threshold offset reduces the efficiency when the ACM mode is switched to a lower one (more protection) because the switch happens earlier than due, i.e. we protect data more than the channel state requires when attenuation is increasing. On the other hand, as mentioned in Section III, when attenuation is decreasing, the delay in changing to a more bandwidth efficient ACM mode introduces an overhead and thus reduces efficiency.

As expected, the threshold offset influences the performance of our technique. The type of rain event - smooth slope or with fluctuations - impacts on PER and efficiency, and a higher $a t t_{\max }$ does not necessary imply worse performance. For example, in Fig. 4, the case of the rain event with $a t t_{\max }=15 \mathrm{~dB}$, which has big fluctuations and thus more ACM mode changes, performs worse than the rain event with $a t t_{\max }=17 \mathrm{~dB}$, which fluctuates less.

Our results generally indicate that a fixed threshold offset is not efficient in terms of bandwidth, e.g. for clear-sky conditions, there is a remarkable loss of efficiency. In other words, the threshold offset that is good for deep fading events, or events with big fluctuations, can not be adequate for low fading or clear sky.

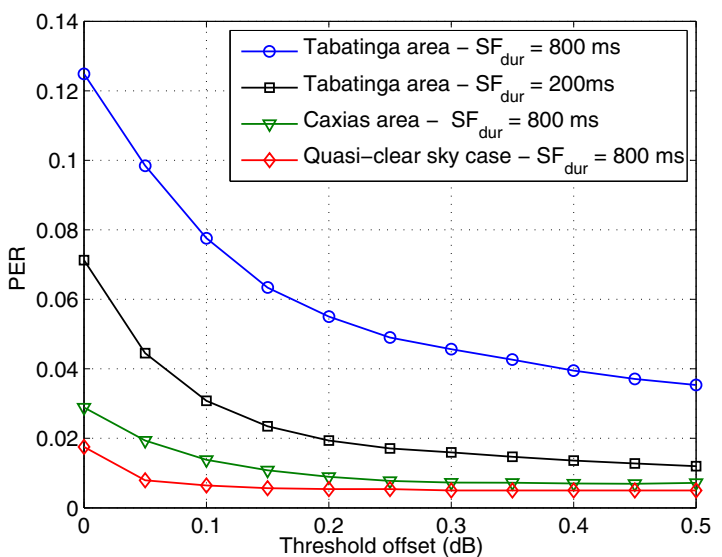

Fig. 3. PER as a function of $t h r_{s}(\mathrm{~dB})$ for several Amazon areas (rain and clear sky conditions), and different super-frame length durations

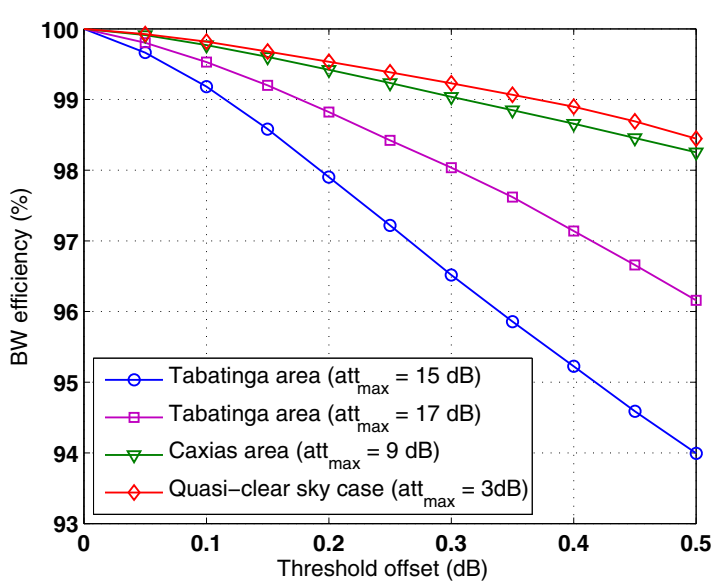

Fig. 4. BW efficiency as a function of $t h r_{s}(\mathrm{~dB})$ for several Amazon areas (rain and clear sky conditions)

\section{Video Quality Evaluation}

In this section the performance of our recovery technique is shown in terms of video quality estimation, by using the traces produced by our simulation to corrupt an MPEG-2 TS (Transport Stream) and comparing it with an uncorrupted TS. We consider the well-known City video samples [6], by evaluating the video quality at the end-user versus the threshold offset. To this end, we compare the video samples received by the end-user with the transmitted original video by using the standard PSNR metric [7]. The PSNR of a picture $P$ is expressed as:

$$
P S N R=10 \log \frac{\left(2^{n}-1\right)^{2}}{M S E(P)}
$$

where $n$ is the number of bits used to represent the luminance of a pixel, and $M S E(P)$ is the Mean Squared Error:

$$
M S E(P)=\frac{1}{N} \sum_{i=1}^{N}\left(P_{i}^{o}-P_{i}^{c}\right)^{2}
$$




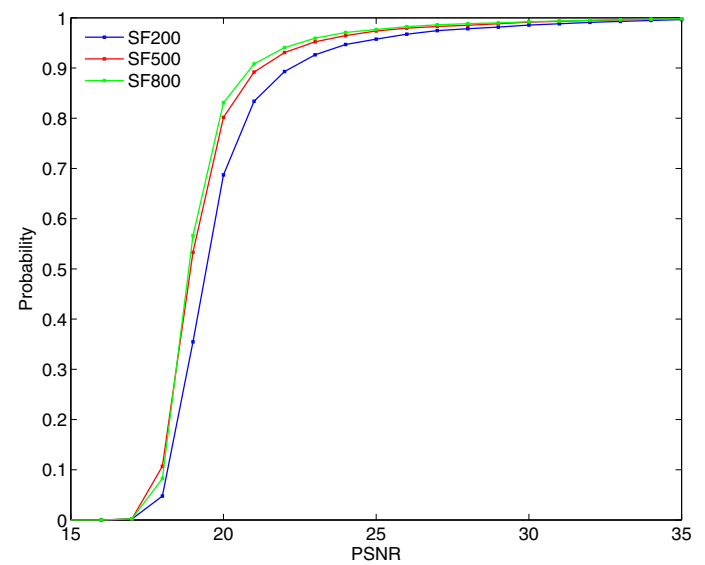

Fig. 5. Cumulative distribution of PSNR.

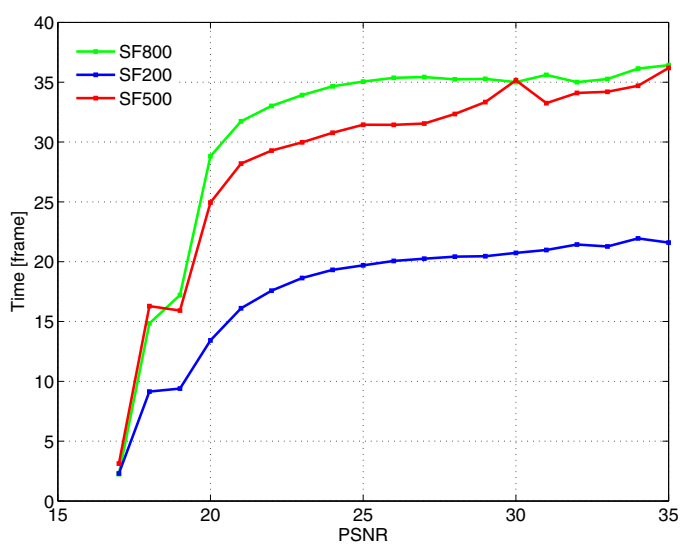

Fig. 6. Average time length of PSNR persistence under a given threshold.

where $N$ is the number of pixels in the picture, while $P^{o}$ and $P^{c}$ and are the original and the corrupted video pictures, respectively.

The PSNR can be a good indicator of the variation of the video quality when the content and codec are fixed across the test conditions [8]. Figure 5 shows the cumulative distribution of the PSNR of the corrupted video for different $S F_{d u r}$ values. Figure 6 shows a second-order statistics of PSNR, that is, the average length of time PSNR $\mathrm{k}$ staying under a given threshold once it crosses it downwards. The figure shows that by fixing a $P S N R_{t h}$ and decreasing the $S F_{d u r}$ length (this means increase the bandwidth overhead) the probability that the video quality is under $P S N R_{t h}$ decreases. Both figures show that the super-frame length has a significant influence on the video quality.

Figure 7 depicts the received video quality versus the threshold offset for two different locations in rain conditions and a quasi-clearsky case. The error traces are the same used for Fig. 3; the performance is evaluated for two $S F_{d u r}$ values. An interesting observation can be made by looking at the quasi-clearsky trace, whose PER is lower than other traces';

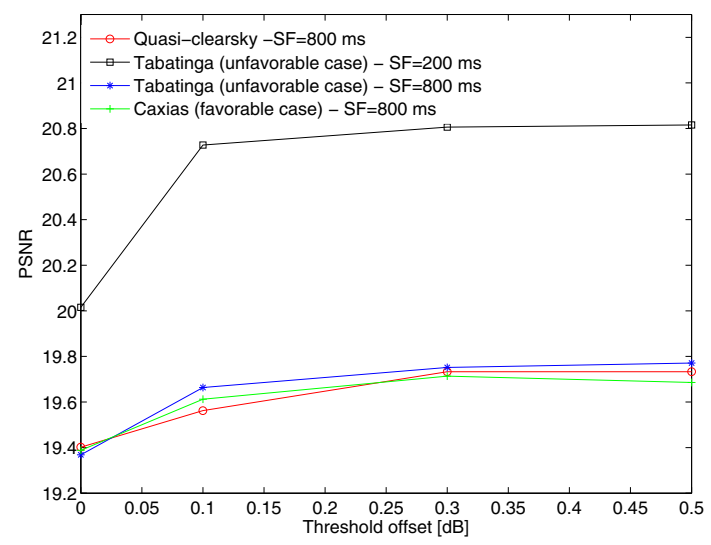

Fig. 7. Mean PSNR versus threshold offset for different Amazon areas and rain types.

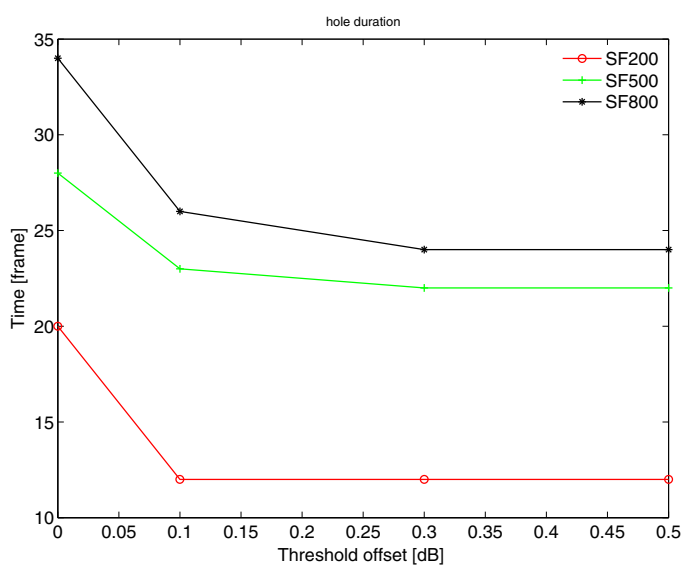

Fig. 8. Mean duration of PSNR corruption depending on the toff and for different $S F_{d u r}$.

however its PSNR is similar to the other traces' when $S F_{d u r}$ is $800 \mathrm{~ms}$. One reason is that the severity of corruption events depend mainly on the number of error bursts, and less on the burst duration.

Figure 8 concentrates on the duration of corruption events, where a corruption event is defined as a sequence of video frames where some video corruption exists. This statistic is insensitive to the threshold offset: there is little difference between setting it to $0.1 \mathrm{~dB}$ or $0.5 \mathrm{~dB}$.

\section{CONCLUSIONS}

This study analyses some performance characteristics of MPEG-2 video streaming over DVB-S2 in the Amazon region. We use the shifted threshold technique for choosing the correct modcod during rain events and make some observations on the video quality as a function of some system parameters.

The DVB-S2 system is emulated by keeping into account realistic allocation delay values and by considering the BBFrame coding. We used the rain events observed during one month in the Amazon region provided by ONERA. 
Results show that a static threshold offset is suboptimal, which means that choosing it dynamically has the potential for significant improvement. The superframe length also has a considerable influence on the video quality, and it should be jointly optimised with the threshold offset. The DVB-S2 technique of histeresys is in principle superior to the simpler shifted offset and would merit a dedicated study.

Future work should additionally consider the trade offs between video quality and channel occupancy.

\section{ACKNOWLEDGEMENTS}

This work was supported in part by the European Commission through SatNEx II (IST-027393), www.satnex.org. We would like to thank the European Commission BRASIL project participants, www.dvb-brasil.org. We thank ONERA for providing us with the tropical attenuation traces. D. Pradas would like also to thank CNES, UAB and TéSA for the joint financing of the research fellowship.

\section{REFERENCES}

[1] H. Skinnemoen, M. A. Vázquez Castro and R. Aroso, Broadband to Rural America over Satellite Integrated Links, Ka and Broadband Communications Conference, Torino 2007.

[2] D. Pradas, P. Barsocchi, L. Jiang, F. Potortì and M. A. Vázquez Castro, Cost-efficient design of hybrid network for video transmission in tropical areas, accepted for publication in IEEE 69th Vehicular Technology Conference (VTC), Barcelona, 2009.

[3] R. Secchi, A. Sathiaseelan, F. Potortì, A. Gotta and G. Fairhurst, Using Quick-Start to enhance TCP-friendly rate control performance in bidirectional satellite networks, Int. J. Commun. Syst. Network, 2009.

[4] ETSI: EN 302307 Digital Video Broadcasting (DVB); Second generation framing structure, channel coding and modulation systems for Broadcasting, Interactive Services, News Gathering and other broadband satellite applications (DVB-S2), June 2006

[5] ETSI: EN 301790 Digital Video Broadcasting (DVB); Interaction Channels for Satellite Distribution Systems, September 2005.

[6] B. Wang, X. Gu, and $\mathrm{H}$. Zhang, An improvement to fine granularity scalability based on h.26l, Circuits and Systems, 2004. ISCAS '04. Proceedings of the 2004 International Symposium on, vol. 3, p. 833, May 2004.

[7] B. G. Haskell and A. N. Netravali, Digital Pictures: Representation, Compression, and Standards, Perseus Publishing, 1997.

[8] Q. Huynh-Thu and M. Ghanbari, Scope of validity of PSNR in image/video quality assessment, in Electronics Letters, vol. 44, Issue 13, pp. 800-801, June 2008. 